\title{
Elset cerrado en voleibol. Diferencias y poder discriminatorio de las acciones finales en etapas de formación \\ Close set in volleyball. Differences and discriminatory power of final game actions in formative stages
}

\author{
Carlos Dávila Romero y Antonio García-Hermoso \\ Universidad de Extremadura
}

\begin{abstract}
Resumen: El objetivo de este estudio fue determinar cuáles de las acciones finales de juego discriminan la victoria y derrota en categorías de formación de voleibol femenino en los sets cerrados resueltos por reglamento con una ventaja mínima de dos puntos. Se analizaron un total de 57 sets en categoría infantil y 69 sets en categoría cadete de los Campeonatos de España de Voleibol de Valladolid 2008 y Huelva 2009 en edad escolar (12-16 años). Los resultados mostraron diferencias entre la condición de ganador y perdedor del set en el ataque positivo en categoría infantil y en el bloqueo positivo y error en categoría cadete. Por su parte, los resultados del análisis discriminante, prueba estadística que determina aquellas acciones de juego más significativas a la hora de establecer diferencias entre los equipos ganadores y perdedores, mostraron como el ataque positivo, servicio negativo y error en categoría infantil y el bloqueo positivo y error en cadete predice la victoria y derrota de set. Estos sugieren que en momentos decisivos de set y en ambas categorías, tanto el control de los gestos técnicos como el error resultante de una infracción reglamentaria, predicen su rendimiento final.
\end{abstract} Palabra clave: Voleibol femenino, análisis notacional, acción de juego, edad escolar.

\begin{abstract}
The aim of this study was to establish which final game actions discriminate final victories and defeats in women volleyball formative stages during ended sets, which are solved by regulation with a minimal advantage of two points. A total of 57 sets were analyzed in infantile category and 69 sets in cadet category during the national volleyball championship at school age (12-16), Valladolid 2008 and Huelva 2009. Statistics analysis shows differences between the condition of both the winner and the loser teams in sets in the positive attack in infantile category and in positive block and error in cadet category. Discriminative analysis, statistical test that determines those most significant game actions when establishing the differences existing between the winner and loser teams, shows how to predict victory and defeat in the ended sets regarding the positive attack, negative service and error in infantile category and positive block and error in cadet category. These ones suggest that during decisive set moments in both categories, either technical gestures control or errors which arise from a regulation infraction may predict their final performance.
\end{abstract}

Key words: Female volleyball, notational analysis, play action, scholar age.

\section{Introducción}

El voleibol es una disciplina deportiva compleja con grandes exigencias técnicas, tácticas y físicas (Kugler, Kruger-Franke, Reininger, Trouillier \& Rosemeyer, 1996), caracterizado por acciones de juego de corta duración y gran intensidad, alternadas por períodos breves de descanso (Künstlinger, Ludwig \& Stegemann, 1987). Elobjetivo general del juego se puede dividir en dos ideas fundamentales, por un lado mantener el saque y por otro, recuperarlo; aunque en la actualidad ambas suponen puntuar, los condicionantes para lograrlo provocan cambios estructurales notables entre una fase y otra (Ureña, Calvo \& Lozano, 2002). Su estructura se divide principalmente en dos complejos de juego: $\mathrm{K} 1$ y K2, donde el primero es conocido como fase de ataque, $\mathrm{y}$ el segundo como fase de defensa. Adicionalmente, otros autores proponen el complejo K3 derivada de la finalización de K1 y K2 (Herrera, Ramos \& Despaigne, 1996), el complejo K4 que corresponde a la fase tras realizar un apoyo o cobertura y por último, el complejo K0 que hace referencia a la acción técnica del saque (Monge, 2003). Así, el final de cada secuencia técnica está estrechamente relacionada con la efectividad de la acción precedente, si bien, aunque las acciones finales son las más relevantes para el rendimiento de un equipo (Moreno, Moreno, García-González, Gil \& del Villar, 2010), las acciones previas inciden en la acción final (Eom, 1989) adquiriendo también importancia estas acciones intermedias.

Los investigadores han estudiado las relaciones entre los diversos factores de juego mediante su análisis, características de los/as jugadores/ as cineantropométricas (Duncan, Woodfield \& Al-Nakeeb, 2006), psicológicas (Stamm, Stamm \& Thomson, 2005) y sus habilidades motoras (Stamm et al., 2003). El análisis de las acciones de juego es una línea de investigación perteneciente al análisis notacional. Son varios los estudios que han utilizado esta metodología en deportes de equipo,

Fecha recepción: 10-05-11 - Fecha envío revisores: 10-05-11 - Fecha de aceptación: 17-06-11 Correspondencia: Antonio García-Hermoso

Avda. Universidad, $\mathrm{s} / \mathrm{n}$.

10.071 Cáceres

E-mail: antoniogh@unex.es como por ejemplo, el baloncesto (Lorenzo, Gómez, Ortega, Ibáñez \& Sampaio, 2010), balonmano (Gruic, Vuleta \& Milanovic, 2006), fútbol (Hughes \& Franks, 2005) y waterpolo (Escalante, Saavedra, Mansilla \& Tella, 2010). Igualmente en voleibol diversos trabajos han utilizado esta metodología a nivel nacional e internacional. En ellos se analizan las acciones técnicas en categoría sénior masculina (Asterios, Kostantinos, Athanasios \& Dimitrios, 2009; Joao, Leite, Mesquita \& Sampaio, 2010; Zetou, Tsigilis, Moustakidis \& Komninakidou, 2006; Zetou, Moustakidis, Tsigilis \& Komninakidou, 2007), existiendo al respecto un menor número de estudios en categoría femenina (Palao, Santos \& Ureña, 2004). En general estudian las estadísticas de juego que predicen la victoria y derrota final de un partido en diferentes campeonatos.

La literatura al respecto muestra un trabajo en el que se investiga las acciones técnicas que discriminan la victoria en cada set (Marelic, Resetar $\&$ Jankovic, 2004). Analizaron concretamente 76 sets de varios partidos de la liga italiana masculina, concluyendo que las variables que discriminaron la victoria en estos sets fueron el remate en fase $\mathrm{K} 1$ y K2, la recepción, el bloqueo y el saque. No obstante, no tuvieron en cuenta las posibles diferencias que podrían existir en los diferentes sets. Estudios en baloncesto confirman que los partidos pueden asumir una estructura diversa en función de la diferencia de puntos (Sampaio, 1998). En este sentido, se ha observado que en los partidos igualados o cerrados, las variables que predicen la victoria y derrota difieren normalmente de los partidos más desequilibrados (Lorenzo et al., 2010). Por lo tanto, parece necesario tener en cuenta las diferencias existentes en función del contexto de juego, si bien ningún estudio en voleibol lo ha tenido en cuenta en sus análisis. Así pues, el objetivo de este estudio fue identificar las acciones finales de juego que discriminan la victoria y derrota de los sets cerrados en categorías de formación de voleibol femenino.

\section{Material y método}

Se recopiló un total de 294 partidos (139 en categoría infantil y 155 en categoría cadete), 1021 sets (478 en categoría infantil y 543 en categoría cadete) y 888 jugadoras (432 en categoría infantil y 456 en categoría cadete). La muestra estuvo compuesta por los resultados y 
estadísticas de los partidos disputados en los Campeonatos de España en edad escolar de Voleibol infantil (12-14 años) y cadete (14-16 años) por Selecciones Autonómicas Femenina, celebrados en Valladolid (2008) y Huelva (2009) (España). Los datos se obtuvieron a través de las páginas web de los campeonatos, mediante las estadísticas oficiales de cada partido, a través del paquete estadístico oficial $\langle$ Infoball», facilitado por la Real Federación Española de Voleibol (RFEVB). De estos datos se tuvieron en cuenta sólo aquellos sets equilibrados que por reglamento se resolvían con una ventaja mínima de dos puntos (FIVB, 2009), analizando un total de 126 sets (57 en categoría infantil y 69 en categoría cadete). Se analizaron estos sets ya que son decisivos en los partidos igualados. De hecho, el 30\% de los partidos a cinco sets se decidieron por la mínima de dos puntos, de los que el $56 \%$ fueron en segunda fase (donde se deciden los primeros puestos del campeonato) y el $11 \%$ en fase final (medallas). El uso de los datos a disposición del público en las páginas web oficiales es habitual en el campo del análisis del rendimiento deportivo en voleibol (Joao, Leite, Mesquita \& Sampaio, 2010).

Se analizaron las siguientes variables:

(i) Ataque positivo: acción técnica ofensiva que conlleva la consecución de un punto.

(ii) Ataque negativo: acción técnica ofensiva que conlleva la pérdida de un punto.

(iii) Saque positivo: acción técnica de saque que conlleva la consecución de un punto.

(iv) Saque negativo: acción técnica de saque que conlleva la pérdida del punto por error directo.

(v) Bloqueo positivo: acción técnica de bloqueo que conlleva la consecución de un punto.

(vi) Error: faltas reglamentarias no delimitadas por alguna de las acciones anteriores que conllevan la pérdida directa del punto.

Se presentaron estadísticos descriptivos, media y desviación típica. Se realizó un análisis exploratorio mediante un test de KolmogorovSmirnov para evaluar la normalidad de la muestra en cada una de las variables, optándose por la realización de pruebas paramétricas al cumplirse la normalidad. Para la comparación entre las medias de los ganadores y perdedores de set se empleó un análisis univariado ANOVA para cada una de las categorías (infantil y cadete). Asimismo, se caleuló el tamaño del efecto (TE) e intervalo de confianza (IC) utilizando las siguientes categorías para su valoración: pequeño si $0 \mathrm{~d} \gg|\mathrm{d}| \mathrm{d} \gg .2$; medio si . $2<|\mathrm{d}| \mathrm{d} » .5 ;$ y largo si $|\mathrm{d}|>.5$ (Cohen, 1988). El método z-scores se utilizó para evaluar la variación de las estadísticas de juego en función de la media de los campeonatos en las distintas categorías y acciones finales.

Posteriormente, se llevo a cabo un análisis discriminante para cada categoría. Este modelo identifica las variables que tienen mayor poder de predicción en el resultado final del set. Se calcularon para cada función el valor Lambda de Wilks, el índice de correlación canónica y el chicuadrado. Se consideraron relevantes para la interpretación de la formula linear los coeficientes estructurales |.300| (Tabachnick \& Fidell, 2007). Se consideró los valores de $p<.05$ estadísticamente significativos. Para todos los análisis de los datos se utilizó el Paquete Estadístico para Ciencias Sociales (SPSS versión 15.0).

\section{Resultados}

En la Tabla 1 se muestran los descriptivos básicos (media y desviación típica) para los dos contextos, set ganado y perdido, así como el análisis univariado ANOVAde las acciones finales de juego para cada categoría (infantil y cadete), el tamaño del efecto (TE) y su intervalo de confianza (IC) en los sets cerrados. Se observa que los equipos ganadores del set presentan valores superiores sólo en el ataque positivo con un TE medio de .40 (95\% IC, .03 a .77) en la categoría infantil. Por su parte, en categoría cadete se observan diferencias entre set ganados y perdidos en el bloqueo positivo y en el error con un TE medio y grande de .38 (95\% IC, .04 a .71) y -.51 (95\% IC, - .84 a -.17), respectivamente.

En la Tabla 2 se muestra el análisis discriminante en cada categoría. En infantil el análisis muestra una función significativa $(p=.05)$, una
Tabla 1. Descriptivos básicos (media y desviación típica), resultados de la comparación de las medias estadísticas, nivel de si gnificación de las variables según el contexto (set ganado y set perdido) y tamaño del efecto ( $95 \%$ intervalo de confianza) en los sets equilibrado

\begin{tabular}{|c|c|c|c|c|c|c|c|c|}
\hline & \multicolumn{2}{|c|}{ Set ganado } & \multicolumn{2}{|c|}{ Set perdido } & \multicolumn{2}{|c|}{ ANOVA } & \multirow{2}{*}{$\mathrm{TE}$} & \multirow{2}{*}{ IC $(95 \%$} \\
\hline & Media & DT & Media & DT & $\mathrm{F}$ & $\mathrm{p}$ & & \\
\hline \multicolumn{9}{|l|}{ Infantil } \\
\hline Ataque positivo (n) & 9.54 & 3.18 & 8.30 & 3.06 & 4.535 & .035 & .40 & .03 а .77 \\
\hline Ataque negativo (n) & 3.74 & 1.65 & 3.42 & 1.89 & 902 & .344 & .18 & -.19 a .55 \\
\hline Saque positi vo (n) & 3.95 & 2.27 & 3.65 & 1.97 & 561 & .455 & .14 & -.23 a .51 \\
\hline Saque negativ o(n) & 2.98 & 1.48 & 3.39 & 1.80 & 1.707 & .194 & -.25 & -62 a .12 \\
\hline Bloqueo positivo (n) & .96 & .84 & 1.05 & 1.26 & .191 & .663 & -08 & -45 a .28 \\
\hline Error $(\mathrm{n})$ & 3.33 & 1.89 & 3.75 & 2.08 & 1.283 & .260 & -.21 & -58 a .16 \\
\hline \multicolumn{9}{|l|}{ Cadete } \\
\hline Ataque positivo (n) & 11.07 & 3.07 & 10.84 & 2.69 & .223 & .638 & .08 & -.25 a .41 \\
\hline Ataque negativo (n) & 3.65 & 2.23 & 4.13 & 1.81 & 1.918 & .168 & -24 & -.57 a .10 \\
\hline Saque positi vo (n) & 2.74 & 1.50 & 2.83 & 1.50 & .115 & .735 & -06 & -.39 a .27 \\
\hline Saque negativ $\mathrm{o}(\mathrm{n})$ & 2.87 & 1.90 & 3.06 & 1.95 & 329 & .567 & -10 & -43 a .24 \\
\hline Bloqueo positivo (n) & 1.98 & 1.47 & 1.42 & 1.51 & 4.967 & .027 & .38 & .04 a .71 \\
\hline Error $(n)$ & 1.78 & 1.27 & 2.51 & 1.60 & 8.645 & .004 & -.51 & $-.84 \mathrm{a}-.17$ \\
\hline
\end{tabular}

DT: desviación típica; TE: tamaño del efecto; IC: intervalo de confianza-

correlación canónica del $.28(\lambda=.91)$ e identifica tres variables predictoras del resultado: el ataque positivo ( $\mathrm{CE}=.669)$, el saque negativo ( $\mathrm{CE}=-$ .410) y el error ( $C E=-.356)$. Por su parte, en categoría cadete, el análisis discriminante muestra una función significativa $(p=.003)$ y una correlación canónica del $.37(\lambda=.86)$, siendo identificados el error $(\mathrm{CE}=-$ .624) y el bloqueo positivo ( $\mathrm{CE}=.473)$ como predictores del resultado de los sets cerrados.

Tabla 2. Análisis discriminante para las diferentes categorías (infantil y cadete) en los sets equilibrados. Se especifica la $\lambda$ Wilks, índice de correlación canónica, chi-cuadrado, nivel de significación y variables seleccionadas en el modelo \begin{tabular}{lcc} 
& Infantil & Cadete \\
\hline$\lambda$ Wilks & .917 & .860 \\
Índice de correlación canónica & .288 & .375 \\
Chi-cuadrado & 14.440 & 20.099 \\
Significación & $\mathbf{. 0 5 0}$ & $\mathbf{. 0 0 3}$ \\
\hline \multirow{2}{*}{ Variables seleccionadas por el modelo } & Ataque positivo & Error \\
& Saque negativo & Bloqueo positivo \\
\hline
\end{tabular} Coeficientes estructurales $|.300|$

\section{Discusión}

El presente estudio ofrece por primera vez datos estadísticos en categoría de formación femenina sobre cuáles fueron las acciones finales de juego que predijeron la victoria en los set cerrados (aquellos que se deciden reglamentariamente por la mínima de dos puntos). Este trabajo aporta información relevante a entrenadores y seleccionadores sobre qué acciones deberían trabajar en momentos igualados de set, así cómo aspectos importantes a planificar en los entrenamientos/concentraciones.

Los equipos que ganan los sets cerrados se diferencian de los equipos perdedores sólo en el ataque positivo en categoría infantil. Se pone de manifiesto el equilibrio existente entre acciones de ataque y defensa para obtener un rendimiento óptimo en estas situaciones de partido. Los resultados constatan que el ataque es una de las acciones finales más determinante para hacer punto, y por lo tanto, resulta una variable determinante del rendimiento como se ha demostrado en diversos estudios en categoría absoluta (Zetou et al., 2007) y de formación (Grgantov, Katic \& Jankovic, 2006). Por su parte, en categoría cadete se observaron diferencias entre set ganado y set perdido en el bloqueo positivo y error, con un tamaño del efecto medio y alto, respectivamente (Tabla 1). El bloqueo positivo, a medida que se produce un desarrollo de las cualidades motrices y cineantropométricas de las jugadoras, va tomando un mayor protagonismo (Stamm et al., 2003). En cuanto a la acción final error, confirma los resultados de diversos estudios que concluyen que existe una relación negativa entre el nivel del equipo, sus jugadores y esta variable (Hughes \& Daniel, 2003; Palao, Santos \& Ureña, 2004).

El análisis discriminante en categoría infantil muestraque las acciones finales que predicen el resultado del set fueron el ataque positivo, el servicio negativo y el error. Respecto al ataque positivo, son varios los estudios que en categoría absoluta han confirmado esta variable como predictora del rendimiento en sus diferentes manifestaciones. Zetou et 
al. (2007) determinaron que en fase K1, el ataque tras salida de recepción, es una de las variables que predice la victoria en jugadores de élite. Del mismo modo, en fase $\mathrm{K} 2$, el ataque tras defensa (contrataque), es otra acción determinante en esta categoría (Häyrinen, Hoivala \& Blomqvist, 2004; Zetou et al., 2006). En este sentido, García y Fernández (2003) analizaron los partidos a tres, cuatro y cinco sets, concluyendo que en estos últimos (a priori más igualados), el ataque es uno de los predictores de la victoria, constatando la importancia del acierto en momentos transcendentales de juego, si bien la muestra analizada en este trabajo difiere en cuanto al género, edad y nivel competitivo. La acción final ataque positivo incluida en el presente análisis, obvia el contexto en el que se produce (toque de antebrazos, toque de dedos, remate, etc.), con lo que no se pueden extraer conclusiones más detalladas al respecto. Probablemente, en estos momentos críticos, el ritmo de juego aumenta, favoreciendo el ataque y a su vez acrecienta los errores en las acciones defensivas (Palao et al., 2004), con lo que el equilibrio entre ambos podría ser una clave del éxito final. No obstante, tanto la literatura existente como los resultados del presente estudio en categoría de formación avalan la importancia de esta acción final en el rendimiento y establece diferencias entre equipos de desigual nivel (Marcelino, Mesquita \& Afonso, 2008).

Otra de las variables seleccionadas en el análisis discriminante, el servicio negativo, confirma los resultados de un trabajo en el que el saque se perfila como un predictor del rendimiento en categoría de formación femenina (12-15 años) (Grgantov et al., 2006). Sin embargo, en el presente estudio a la inversa, es decir, obtener un mayor número de errores en el servicio predice la derrota en el set. En este sentido, otro trabajo reciente en categoría de formación femenina determina que la relación entre error-acierto (punto directo) en el servicio es de 1:1 (Gil, Moreno, Moreno, García \& Del Villar, 2011), por lo tanto, parece lógico que los errores propios no forzados es un factor que debe regularse durante el entrenamiento y la competición, pudiendo disminuir así su incidencia (Häyrinen et al., 2004). En categoría infantil, al igual que se ha observado en otras categorías superiores y presuntamente con un mayor control técnico (cadete y juvenil), no se aprecia un dominio y control del servicio suficiente como para evidenciar una intencionalidad táctica clara, donde las jugadoras asumirían un nivel de riesgo voluntario y adaptado a la situación de partido, aspecto caracterizador del presente trabajo (García-Tormo, Redondo, Vallares \& Morante, 2006). Esta situación se observa del mismo modo en tenis, el jugador novel pese a disponer del mismo tiempo que el experto para la realización del saque, no tiene el mismo grado de conciencia sobre las variables contextuales de eficacia (Del Villar, García, Iglesias, Moreno \& Cervelló, 2007). Por lo tanto, estos resultados parecen indicar que aquellos equipos que obtengan un mayor acierto y control en el patrón técnico del saque tienen más probabilidades de ganar los sets cerrados.

En ambas categorías, el análisis discriminante seleccionó como acción final que predice la derrota el error. En este sentido, García-Tormo et al. (2006) observaron en categoría cadete y juvenil, la existencia de una relación entre el nivel de riesgo asumido y los puntos restantes para el final de set. Por lo tanto, la tensión emocional, el estrés competitivo y la carga física experimentan un notable incremento en los puntos finales de cada set, lo que parece ser determinante en estos momentos de juego, aspecto que se ha corroborado en baloncesto (Labrador, Crespo, Buceta \& González, 1995). Parece lógico pensar que el error en partidos y sets cerrados sea una de las acciones determinantes de la derrota, más si cabe en categorías de formación. Se ha confirmado que los jugadores noveles cometen más errores que los jugadores expertos (Hughes \& Daniel, 2003), tendencia que se observa en el presente trabajo entre equipos ganadores y perdedores del set, especialmente en categoría cadete. De este modo, el entrenador podría trabajar en la reducción del número de errores cometidos en cada partido y con ello aumentar las posibilidades del equipo de ganar el set.

Por último, en categoría cadete, se observa el bloqueo positivo como acción final determinante de los set cerrados. El estudio de Marelic et al. (2004) confirman esta acción de juego como discriminante de la victoria de set en categoría absoluta masculina de élite. Por lo tanto, se corrobora la importancia de las acciones cercanas a la red como predictoras de rendimiento (Stamm et al., 2003). Dominar esta acción técnica defensiva (fase K2) permitirá a los equipos llegar con garantías de victoria a los finales decisivos de set. En este sentido, un estudio analizó a 197 jugadoras de 14-17 años, mostrando que la técnica de bloqueo es una de las variables más relevantes de juego (Katic, Grgantov \& Jurko, 2006). Aquellos equipos cuyas jugadoras sean capaces de tomar mejores decisiones a la hora de realizar el movimiento de bloqueo y ejecutarlo eficazmente, tendrán más posibilidades de ganar un set cerrado (McPherson, Dovenmuheler \& Murray, 1992). En voleibol, el tomar decisiones en el menor tiempo posible denota un carácter importante y fundamental para un mayor rendimiento (Moreno et al., 2010).

\section{Conclusiones}

Las conclusiones que se pueden extraer del presente estudio fueron: (i) en los sets cerrados (dos puntos de diferencia) el ataque positivo diferencia a los equipos ganadores de los perdedores en categoría infantil, siendo en categoría cadete el bloqueo positivo y el error; (ii) las acciones finales que predicen la victoria en los sets cerrados son el ataque positivo, servicio negativo yel error en categoría infantil, evidenciando la importancia del acierto en acciones ofensivas como en el control en el patrón técnico del saque en momentos de sets igualados y trascendentales; y (iii) en categoría cadete las acciones finales que discriminan entre ganadores y perdedores en los sets cerrados son el error y el bloqueo positivo, lo que parece indicar que a medida que se produce el desarrollo madurativo de las jugadoras, las acciones discriminantes en estas situaciones van dirigidas a técnicas más complejas cercanas a la red como el bloqueo, así como un mayor dominio técnico que propicie la ausencia de errores.

Conocer las estadísticas de juego y su capacidad de predicción de la victoria y derrota en categorías de formación, permitirá a los entrenadores/seleccionadores tener datos objetivos sobre las acciones técnicas y el riesgo a asumir por las jugadoras, lo que puede aportar un mejor planteamiento ante futuras situaciones de igualdad competitiva. No obstante, los datos oficiales que recogen estos campeonatos a través del «Infoball» se ciñen tan sólo a acciones finales de juego, por lo que no permite analizar el peso específico que confieren acciones técnicas intermedias durante el partido. Sería interesante realizar estudios en estas categorías a través de estadísticas más concretas y detalladas mediante programas más asentados en el panorama internacional como el «Data Volley». Del mismo modo, se podría tener en cuenta el número de set de cada partido (tres, cuatro o cinco sets), fase del torneo (primera fase, segunda fase y final) y diferencia entre géneros (masculino y femenino).

\section{Agradecimientos}

Al Prof. Dr. D. José M. Saavedra, por su valioso aporte en la revisión de este documento.

\section{Referencias}

Asterios, P., Kostantinos, C., Athanasios, M., \& Dimitrios, K. (2009). Comparison of technical skills effectiveness of men's National Volleyball teams. International Journal of Performance Analysis of Sport, 9(1), 1-7.

Cohen, J.(1988). Statistical Power Analysisfor the Behavioral Sciences Hillsdale. New Jersey: Lawrence Erlbaum Associates.

Del Villar, F., García, L., Iglesias, D., Moreno, M. P., \& Cervelló, E. M. (2007). Expert-novice differences in cognitive and execution skills during tennis competition. Perceptual and Motor Skills, 104, 355365 .

Duncan, M. J., Woodfield, L., \& al-Nakeeb, Y. (2006). Anthropometric and physiological characteristics of junior elite volleyball players. British Journal of Sports Medicine, 40(7), 649-651. 
Escalante, Y., Saavedra, J. M., Mansilla, M., \& Tella, V. Discriminatory power of water polo game-related statistics at the 2008 Olympic Games. Journal of Sports Science, 29(3), 291-298.

Eom, H.J. (1989). Computer-aided recording and Mathematical analysis of team perfomance in volleyball. Disertación doctoral no publicada, The British Columbia University, Vancouver, Canadá.

FIVB. (2009). Reglas oficiales de voleibol. Recuperado de www.rfevb.com

García, F. A., \& Fernández, F. (2003). Acciones determinantes en el resultado en voleibol. Congreso Internacional sobre Entrenamiento Deportivo. Valladolid.

García-Tormo, J. V.; Redondo J. C.; Valladares, J. A., \& Morante, J. C. (2006). Análisis del saque de voleibol categoría juvenil femenina en función nivel de riesgo asumido y su eficacia. European Journal of Human Movement. Motricidad, 16, 99-121.

Gil, A., Moreno, M. P., Moreno, A., García, L., \& Del Villar, F. (2011). Estudio del saque en jóvenes jugadores/as de voleibol, considerando la eficaciay función en juego. Retos. Nuevastendencias en Educación Física, Deporte y Recreación, 19, 19-24.

Grgantov, Z, Katic, R., \& Jankovic, V. (2006). Morphological Characteristics, Technical and Situation Efficacy of Young Female Volleyball Players. Collegium Antropologicum, 30(1), 87-96.

Gruic, I., Vuleta, D., \& Milanovic, D. (2006). Performance indicators of teams at the 2003 Men's World Handball Championship in Portugal. Kinesiology, 38(2), 164-175.

Häyrinen, M., Hoivala, T., \& Blomqvist M. (2004). Differences between winning and losing teams in men's European top-level volleyball. En: P. O'Donoghue \& M. Hughes (Ed.), Performance Analysis of Sport VI (pp. 194-199). Cardiff: UWIC.

Hughes, M., \& Daniel, R. (2003). Playing patterns of elite and non-elite volleyball. International Journal of Performance Analysis in Sport, $3(1), 50-56$.

Hughes, M., \& Franks, I. (2005). Analysis of passing sequences, shot and goals in soccer. Journal of Sports Sciences, 23(5), 509-514.

Herrera, G., Ramos, J., \& Despaigne, J. (1996). Voleibol: Manual de consulta operativa para el entrenador. Federación Vasca de Voleibol.

Katic, R., Grgantov, Z., \& Jurko, D. (2006). Motor Structures in Female Volleyball Players Aged 14-17 According to Technique Quality and Performance. Collegium Antropologicum, 30(1), 103-112.

Kugler, A., Kruger-Franke, M., Reininger, S., Trouillier, H. H., \& Rosemeyer, B. (1996). Muscular imbalance and shoulder pain in volleyball attackers. British Journal of Sports Medicine, 30(3), 256-259.

Kunstlinger, U., Ludwig, H. G., \& Stegemann, J. (1987). Metabolic changes during volleyball matches. International Journal Sports Medicine, 8(5), 315-322.

Joao, P. V., Leite, N., Mesquita, I., \& Sampaio, J. (2010). Sex differences in discriminative power of volleyball game-related statistics. Perceptual and Motor Skills, 111(3), 893-900.

Labrador, F. J., Crespo, M., Buceta, J. M., \& González, S. (1995). Factores contextuales implicados en el lanzamiento de tiros libres en baloncesto. Revista de Psicología del Deporte, 7, 87-89.
Lorenzo, A., Gómez, M. A., Ortega, E., Ibáñez, S. J., \& Sampaio, J. (2010). Game related statistics which discriminate between winning and losing under-16 male basketball games. Journal of Sports Science and Medicine, 9, 664-668.

Marcelino, R., Mesquita, I., \& Afonso, J. (2008) The weight of terminal actions in Volleyball. Contributions of the spike, serve and block for the teams' rankings in the World League'2005. International Journal of Performance Analysis in Sport, 88(2), 1-7.

Marelić, N., Resetar, T., \& Janković, V. (2004). Discriminant analysis of the sets won and the sets lost by one team in A1 Italian volleyball league - A case study. Kinesiology, 36, 75-82.

McPherson, S. L., Dovenmuheler, A., \& Murray, M. (1992). Player differences in representation of strategic knowledge and use during a modified volleyball blocking game situation. Paper presentado en Meeting of the North American Society for the Psychology of Sport and Physical Activity, Pittsburgh, PA.

Monge, A. M. (2003). Propuesta estructural del desarrollo del juego en Voleibol. En: I. Mesquita, C. A. S. S. Mountinho, C.A.S.S., \& R. Faria (Eds.). Investigação em voleibol: estudos ibéricos (pp. 142149). Porto: FCDEF-UP.

Moreno, A., Moreno, M. P., García-González, L., Gil, A., \& Del Villar, F. (2010). La importancia de la función en juego en la representación de problemas en jugadoras de voleibol noveles. Kronos, 17, 65-72.

Palao, J. M., Santos, J. A., \& Ureña, A. (2004). Effect of team level on skill performance in volleyball. International Journal of Performance Analysis of Sport, 4(2), 50-60.

Sampaio, J. (1998). Los indicadores estadisticos mas determinantes en el resultado final en los partidos de basquetbol. Lecturas: Revista Digital de Educácion Física y Deporte, 11.

Stamm, R., Stamm, M., \& Thomson, K. (2005). Role of adolescent female volleyball players' psychophysiological properties and body build in performance of different elements of the game. Perceptual and Motor Skills, 101(1), 108-120.

Stamm, R., Veldre, G., Stamm, M., Thomson, K., Kaarma, H., Loko, J., et al. (2003). Dependence of young female volleyballer's performance on their body build, physical abilities, and psychophysiological properties. Journal of Sports Medicine and Physical Fitness, 43(3), 291-299.

Tabachnick, B., \& Fidell, L. (2007). Using multivariate statistics (5th ed.). New York: Harper and Row Publishers.

Ureña, A., Calvo, R. M., \& Lozano, C. (2002). Estudio de la recepción del saque en el voleibol masculino español de elite tras la incorporación del jugador libero. Revista Internacional de Medicina y Ciencias de la Actividad Física y el Deporte, 2(4), 37-49.

Zetou, E., Tsiggilis, N., Moustakidis, A., \& Komninakidou, A. (2006). Playing characteristics of men's Olympic Volleyball teams in complex II. International Journal of Performance Analysis in Sport, 6(1), 172-177.

Zetou, E., Moustakidis, A., Tsiggilis, N., \& Komninakidou, A. (2007). «Does Effectiveness of Skill in Complex I Predict Win in Men's Olympic Volleyball Games? Journal of Quantitative Analysis in Sports, 3(4), article 3.

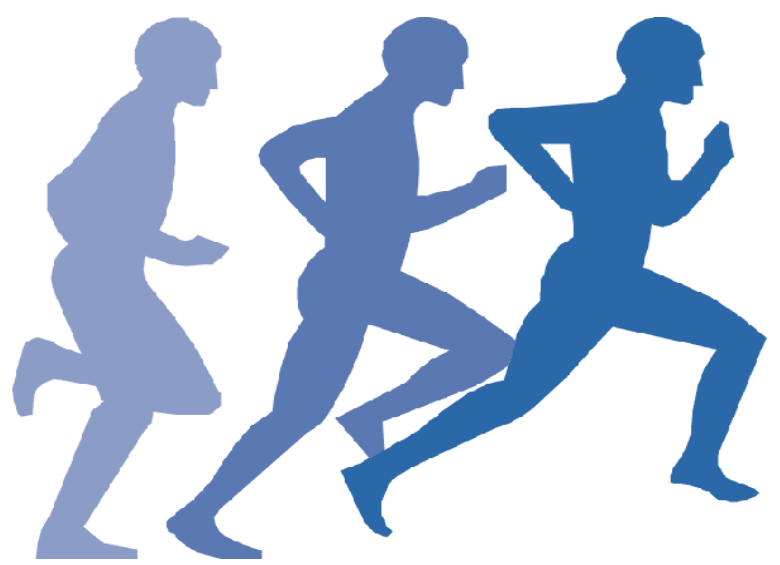

